\title{
On the tripled fixed point and tripled coincidence point theorems in fuzzy normed spaces
}

\author{
Reza Saadati ${ }^{1}$ Poom Kumam² and Sun Young Jang ${ }^{3 *}$
}

"Correspondence: jsym@ulsan.ac.kr ${ }^{3}$ Department of Mathematics, University of Ulsan, Ulsan, 680-749, South Korea

Full list of author information is available at the end of the article

\begin{abstract}
Recently, Abbas et al. (Fixed Point Theory Appl. 2012:187, 2012) proved tripled fixed point and tripled coincidence point theorems in intuitionistic fuzzy normed spaces. Saadati and Park proved that the topology $\tau_{(\mu, \nu)}$ generated by an intuitionistic fuzzy normed space $(X, \mu, \nu, *, \diamond)$ coincides with the topology $\tau_{\mu}$ generated by the generalized fuzzy normed space $(X, \mu, *)$, and thus the results obtained in intuitionistic fuzzy normed spaces are immediate consequences of the corresponding results for fuzzy normed spaces. In this paper, we improve and extend the results presented by Abbas et al. to $\mathcal{L}$-fuzzy normed spaces.
\end{abstract}

MSC: $47 \mathrm{H} 09 ; 47 \mathrm{H} 10 ; 54 \mathrm{H} 25$

Keywords: $\mathcal{L}$-fuzzy set; INFS; $t$-norm and $t$-conorm; tripled fixed point; tripled coincidence point

\section{Introduction}

Intuitionistic fuzzy normed spaces were investigated by Saadati and Park [1]. They introduced and studied intuitionistic fuzzy normed spaces based both on the idea of intuitionistic fuzzy sets due to Atanassov [2] and the concept of fuzzy normed spaces given by Saadati and Vaezpour in [3]. In [4] Saadati and Park proved that the topology $\tau_{(\mu, v)}$ generated by an intuitionistic fuzzy normed space $(X, \mu, \nu, *, \diamond)$ coincides with the topology $\tau_{\mu}$ generated by the generalized fuzzy normed space $(X, \mu, *)$, and thus the results obtained in intuitionistic fuzzy normed spaces are immediate consequences of the corresponding results for fuzzy normed spaces. For improving this problem, Deschrijver et al. [5] modified the concept of intuitionistic fuzzy normed spaces and introduced the notation of $\mathcal{L}$-fuzzy normed space.

Fixed point theorems have been studied in many contexts, one of which is the fuzzy setting. The concept of fuzzy sets was initially introduced by Zadeh [6] in 1965. To use this concept in topology and analysis, many authors have extensively developed the theory of fuzzy sets and its applications. One of the most interesting research topics in fuzzy topology is to find an appropriate definition of fuzzy metric space for its possible applications in several areas. It is well known that a fuzzy metric space is an important generalization of the metric space. Many authors have considered this problem and have introduced it in different ways. For instance, George and Veeramani [7] modified the concept of a fuzzy metric space introduced by Kramosil and Michalek [8] and defined the Hausdorff topology 
of a fuzzy metric space. There exists considerable literature about fixed point properties for mappings defined on fuzzy metric spaces, which have been studied by many authors (see [9-13]). Zhu and Xiao [14] and $\mathrm{Hu}$ [15] gave a coupled fixed point theorem for contractions in fuzzy metric spaces (see also [16]). Recently, Abbas et al. [17] proved tripled fixed point and tripled coincidence point theorems in intuitionistic fuzzy normed spaces. In this paper we present some shorter proofs for Abbas et al.'s results in $\mathcal{L}$-fuzzy normed space, which is not a trivial generalization of fuzzy normed space.

\section{Fuzzy normed spaces}

A binary operation $*:[0,1] \times[0,1] \rightarrow[0,1]$ is a continuous $t$-norm if it satisfies the following conditions:

(a) $*$ is associative and commutative;

(b) $*$ is continuous;

(c) $a * 1=a$ for all $a \in[0,1]$;

(d) $a * b \leq c * d$ whenever $a \leq c$ and $b \leq d$, for each $a, b, c, d \in[0,1]$.

Two typical examples of continuous $t$-norm are $a * b=a b$ and $a * b=\min (a, b)$.

A binary operation $\diamond:[0,1] \times[0,1] \rightarrow[0,1]$ is a continuous $t$-conorm if it satisfies the following conditions:

(a) $\diamond$ is associative and commutative;

(b) $\diamond$ is continuous;

(c) $a \diamond 0=a$ for all $a \in[0,1]$;

(d) $a \diamond b \leq c \diamond d$ whenever $a \leq c$ and $b \leq d$, for each $a, b, c, d \in[0,1]$.

Two typical examples of continuous $t$-conorm are $a \diamond b=\min (a+b, 1)$ and $a \diamond b=$ $\max (a, b)$.

In 2005, Saadati and Vaezpour [3] introduced the concept of fuzzy normed spaces.

Definition 2.1 Let $X$ be a real vector space. A function $\mu: X \times \mathbb{R} \rightarrow[0,1]$ is called a fuzzy norm on $X$ if for all $x, y \in X$ and all $s, t \in \mathbb{R}$ :

( $\left.\mu_{1}\right) \quad \mu(x, t)=0$ for $t \leq 0$;

$\left(\mu_{2}\right) x=0$ if and only if $\mu(x, t)=1$ for all $t>0$;

$\left(\mu_{3}\right) \mu(c x, t)=\mu\left(x, \frac{t}{|c|}\right)$ if $c \neq 0$

$\left(\mu_{4}\right) \mu(x+y, s+t) \geq \mu(x, s) * \mu(y, t)$;

$\left(\mu_{5}\right) \quad \mu(x, \cdot)$ is a non-decreasing function of $\mathbb{R}$ and $\lim _{t \rightarrow \infty} \mu(x, t)=1$;

$\left(\mu_{6}\right)$ for $x \neq 0, \mu(x, \cdot)$ is continuous on $\mathbb{R}$.

For example, if $a * b=a b$ for $a, b \in[0,1],(X,\|\cdot\|)$ is a normed space and

$$
\mu(x, t)=\frac{t}{t+\|x\|}
$$

for all $x, y, z \in X$ and $t>0$. Then $\mu$ is a (standard) fuzzy normed and $(X, \mu, \cdot)$ is a fuzzy normed space.

Saadati and Vaezpour showed in [3] that every fuzzy norm $(\mu, *)$ on $X$ generates a first countable topology $\tau_{\mu}$ on $X$ which has as a base the family of open sets of the form $\left\{B_{\mu}(x, r, t): x \in X, r \in(0,1), t>0\right\}$ where $B_{\mu}(x, r, t)=\{y \in X: \mu(x-y, t)>1-r\}$ for all $x \in X$, $r \in(0,1)$ and $t>0$. 


\section{Intuitionistic fuzzy normed spaces}

Saadati and Park [1] defined the notion of intuitionistic fuzzy normed spaces with the help of continuous $t$-norms and continuous $t$-conorms as a generalization of fuzzy normed space due to Saadati and Vaezpour [3].

Definition 3.1 The 5-tuple $(X, \mu, v, *, \diamond)$ is said to be an intuitionistic fuzzy normed space if $X$ is a vector space, $*$ is a continuous $t$-norm, $\diamond$ is a continuous $t$-conorm, and $\mu, v$ are fuzzy sets on $X \times(0, \infty)$ satisfying the following conditions for every $x, y \in X$ and $t, s>0$ :
(a) $\mu(x, t)+v(x, t) \leq 1$
(b) $\mu(x, t)>0$;
(c) $\mu(x, t)=1$ if and only if $x=0$;
(d) $\mu(\alpha x, t)=\mu\left(x, \frac{t}{|\alpha|}\right)$ for each $\alpha \neq 0$;
(e) $\mu(x, t) * \mu(y, s) \leq \mu(x+y, t+s)$;
(f) $\mu(x, \cdot):(0, \infty) \rightarrow[0,1]$ is continuous;
(g) $\lim _{t \rightarrow \infty} \mu(x, t)=1$ and $\lim _{t \rightarrow 0} \mu(x, t)=0$;
(h) $v(x, t)<1$;
(i) $v(x, t)=0$ if and only if $x=0$;
(j) $v(\alpha x, t)=v\left(x, \frac{t}{|\alpha|}\right)$ for each $\alpha \neq 0$;
(k) $v(x, t) \diamond v(y, s) \geq v(x+y, t+s)$;
(l) $v(x, \cdot):(0, \infty) \rightarrow[0,1]$ is continuous;
(m) $\lim _{t \rightarrow \infty} v(x, t)=0$ and $\lim _{t \rightarrow 0} v(x, t)=1$.

In this case $(\mu, v)$ is called an intuitionistic fuzzy norm.

Example 3.2 Let $(X,\|\cdot\|)$ be a normed space. Denote $a * b=a b$ and $a \diamond b=\min (a+b, 1)$ for all $a, b \in[0,1]$ and let $\mu$ and $v$ be fuzzy sets on $X \times(0, \infty)$ defined as follows:

$$
\mu(x, t)=\frac{t}{t+\|x\|}, \quad v(x, t)=\frac{\|x\|}{t+\|x\|}
$$

for all $t \in \mathbf{R}^{+}$. Then $(X, \mu, v, *, \diamond)$ is an intuitionistic fuzzy normed space.

Saadati and Park proved in [1] that every intuitionistic fuzzy norm $(\mu, \nu)$ on $X$ generates a first countable topology $\tau_{(\mu, v)}$ on $X$ which has as a base the family of open sets of the form $\left\{B_{(\mu, v)}(x, r, t): x \in X, r \in(0,1), t>0\right\}$ where $B_{(\mu, v)}(x, r, t)=\{y \in X: \mu(x-y, t)>1-r, v(x-$ $y, t)<r\}$ for all $x \in X, r \in(0,1)$ and $t>0$.

Lemma 3.3 ([4]) Let $(X, \mu, \nu, *, \diamond)$ be an intuitionistic fuzzy normed space. Then, for each $x \in X, r \in(0,1)$ and $t>0$ we have $B_{(\mu, v)}(x, r, t)=B_{\mu}(x, r, t)$.

From Lemma 3.3, we deduce the following.

Theorem 3.4 Let $(X, \mu, v, *, \diamond)$ be an intuitionistic fuzzy normed space. Then the topologies $\tau_{(\mu, \nu)}$ and $\tau_{\mu}$ coincide on $X$.

\section{$4 \mathcal{L}$-Fuzzy normed spaces}

Definition 4.1 ([18]) Let $\mathcal{L}=\left(L, \leq_{L}\right)$ be a complete lattice and $U$ a non-empty set called universe. An $\mathcal{L}$-fuzzy set $\mathcal{A}$ on $U$ is defined as a mapping $\mathcal{A}: U \rightarrow L$. For each $u$ in $U, \mathcal{A}(u)$ represents the degree (in $L$ ) to which $u$ satisfies $\mathcal{A}$. 
Lemma 4.2 ([19]) Consider the set $L^{*}$ and operation $\leq_{L^{*}}$ defined by

$$
L^{*}=\left\{\left(x_{1}, x_{2}\right):\left(x_{1}, x_{2}\right) \in[0,1]^{2} \text { and } x_{1}+x_{2} \leq 1\right\},
$$

$\left(x_{1}, x_{2}\right) \leq_{L^{*}}\left(y_{1}, y_{2}\right) \Longleftrightarrow x_{1} \leq y_{1}$ and $x_{2} \geq y_{2}$, for every $\left(x_{1}, x_{2}\right),\left(y_{1}, y_{2}\right) \in L^{*}$. Then $\left(L^{*}, \leq_{L^{*}}\right)$ is a complete lattice.

Definition 4.3 ([2]) An intuitionistic fuzzy set $\mathcal{A}_{\zeta, \eta}$ on a universe $U$ is an object $\mathcal{A}_{\zeta, \eta}=$ $\left\{\left(\zeta_{\mathcal{A}}(u), \eta_{\mathcal{A}}(u)\right): u \in U\right\}$, where, for all $u \in U, \zeta_{\mathcal{A}}(u) \in[0,1]$ and $\eta_{\mathcal{A}}(u) \in[0,1]$ are called the membership degree and the non-membership degree, respectively, of $u$ in $\mathcal{A}_{\zeta, \eta}$, and they furthermore satisfy $\zeta_{\mathcal{A}}(u)+\eta_{\mathcal{A}}(u) \leq 1$.

Classically, a triangular norm $T$ on $([0,1], \leq)$ is defined as an increasing, commutative, associative mapping $T:[0,1]^{2} \rightarrow[0,1]$ satisfying $T(1, x)=x$, for all $x \in[0,1]$. These definitions can be straightforwardly extended to any lattice $\mathcal{L}=\left(L, \leq_{L}\right)$. Define first $0_{\mathcal{L}}=\inf L$ and $1_{\mathcal{L}}=\sup L$.

Definition 4.4 A triangular norm ( $t$-norm) on $\mathcal{L}$ is a mapping $\mathcal{T}: L^{2} \rightarrow L$ satisfying the following conditions:

(i) $(\forall x \in L)\left(\mathcal{T}\left(x, 1_{\mathcal{L}}\right)=x\right)$ (boundary condition);

(ii) $\left(\forall(x, y) \in L^{2}\right)(\mathcal{T}(x, y)=\mathcal{T}(y, x))$ (commutativity);

(iii) $\left(\forall(x, y, z) \in L^{3}\right)(\mathcal{T}(x, \mathcal{T}(y, z))=\mathcal{T}(\mathcal{T}(x, y), z))$ (associativity);

(iv) $\left(\forall\left(x, x^{\prime}, y, y^{\prime}\right) \in L^{4}\right)\left(x \leq_{L} x^{\prime}\right.$ and $\left.y \leq_{L} y^{\prime} \Longrightarrow \mathcal{T}(x, y) \leq_{L} \mathcal{T}\left(x^{\prime}, y^{\prime}\right)\right)$ (monotonicity).

A $t$-norm can also be defined recursively as an $(n+1)$-ary operation $(n \in \mathbf{N} \backslash\{0\})$ by $\mathcal{T}^{1}=\mathcal{T}$ and

$$
\mathcal{T}^{n}\left(x_{(1)}, \ldots, x_{(n+1)}\right)=\mathcal{T}\left(\mathcal{T}^{n-1}\left(x_{(1)}, \ldots, x_{(n)}\right), x_{(n+1)}\right)
$$

for $n \geq 2$ and $x_{(i)} \in L$.

The $t$-norm $\mathbf{M}$ defined by

$$
\mathbf{M}(x, y)= \begin{cases}x & \text { if } x \leq_{L} y \\ y & \text { if } y \leq_{L} x\end{cases}
$$

is a continuous $t$-norm.

Definition 4.5 ([20]) A $t$-norm $\mathcal{T}$ on $L^{*}$ is called $t$-representable if and only if there exist a $t$-norm $T$ and a $t$-conorm $S$ on $[0,1]$ such that, for all $x=\left(x_{1}, x_{2}\right), y=\left(y_{1}, y_{2}\right) \in L^{*}$,

$$
\mathcal{T}(x, y)=\left(T\left(x_{1}, y_{1}\right), S\left(x_{2}, y_{2}\right)\right) .
$$

Definition 4.6 A negation on $\mathcal{L}$ is any strictly decreasing mapping $\mathcal{N}: L \rightarrow L$ satisfying $\mathcal{N}\left(0_{\mathcal{L}}\right)=1_{\mathcal{L}}$ and $\mathcal{N}\left(1_{\mathcal{L}}\right)=0_{\mathcal{L}}$. If $\mathcal{N}(\mathcal{N}(x))=x$, for all $x \in L$, then $\mathcal{N}$ is called an involutive negation.

In this paper, $\mathcal{N}: L \rightarrow L$ is fixed. The negation $N_{s}$ on $([0,1], \leq)$ defined, for all $x \in[0,1]$, by $N_{s}(x)=1-x$, is called the standard negation on $([0,1], \leq)$. 
Definition 4.7 The 3-tuple $(X, \mathcal{P}, \mathcal{T})$ is said to be an $\mathcal{L}$-fuzzy normed space if $X$ is a vector space, $\mathcal{T}$ is a continuous $t$-norm on $\mathcal{L}$ and $\mathcal{P}$ is an $\mathcal{L}$-fuzzy set on $X \times] 0,+\infty$ [ satisfying the following conditions for every $x, y$ in $X$ and $t, s$ in $] 0,+\infty[$ :

(a) $0_{\mathcal{L}}<L$

(b) $\mathcal{P}(x, t)=1_{\mathcal{L}}$ if and only if $x=0$;

(c) $\mathcal{P}(\alpha x, t)=\mathcal{P}\left(x, \frac{t}{|\alpha|}\right)$ for each $\alpha \neq 0$;

(d) $\mathcal{T}(\mathcal{P}(x, t), \mathcal{P}(y, s)) \leq{ }_{L} \mathcal{P}(x+y, t+s)$;

(e) $\mathcal{P}(x, \cdot):] 0, \infty[\rightarrow L$ is continuous;

(f) $\lim _{t \rightarrow 0} \mathcal{P}(x, t)=0_{\mathcal{L}}$ and $\lim _{t \rightarrow \infty} \mathcal{P}(x, t)=1_{\mathcal{L}}$.

In this case $\mathcal{P}$ is called an $\mathcal{L}$-fuzzy norm. If $\mathcal{P}=\mathcal{P}_{\mu, v}$ is an intuitionistic fuzzy set and the $t$-norm $\mathcal{T}$ is $t$-representable, then the 3 -tuple $\left(X, \mathcal{P}_{\mu, v}, \mathcal{T}\right)$ is said to be an intuitionistic fuzzy normed space.

Definition 4.8 A sequence $\left\{x_{n}\right\}_{n \in \mathbf{N}}$ in an $\mathcal{L}$-fuzzy normed space $(X, \mathcal{P}, \mathcal{T})$ is called a Cauchy sequence if for each $\varepsilon \in L \backslash\left\{0_{\mathcal{L}}\right\}$ and $t>0$, there exists $n_{0} \in \mathbf{N}$ such that

$$
\mathcal{N}(\varepsilon)<_{L} \mathcal{P}\left(x_{n}-x_{m}, t\right)
$$

for each $n, m \geq n_{0}$; here $\mathcal{N}$ is an involutive negation. The sequence $\left\{x_{n}\right\}_{n \in \mathbf{N}}$ is said to be convergent to $x \in X$ in the $\mathcal{L}$-fuzzy normed space $(X, \mathcal{P}, \mathcal{T})$ and denoted by $x_{n} \stackrel{\mathcal{P}}{\rightarrow} x$ if $\mathcal{P}\left(x_{n}-\right.$ $x, t) \rightarrow 1_{\mathcal{L}}$ whenever $n \rightarrow+\infty$ for every $t>0$. An $\mathcal{L}$-fuzzy normed space is said to be complete if and only if every Cauchy sequence is convergent.

Lemma 4.9 ([21]) Let $\mathcal{P}$ be an $\mathcal{L}$-fuzzy norm on $X$. Then:

(i) $\mathcal{P}(x, t)$ is non-decreasing with respect to $t$, for all $x$ in $X$;

(ii) $\mathcal{P}(x-y, t)=\mathcal{P}(y-x, t)$, for all $x, y$ in $X$ and $t \in] 0,+\infty[$.

Definition 4.10 Let $(X, \mathcal{P}, \mathcal{T})$ be an $\mathcal{L}$-fuzzy normed space. For $t \in] 0,+\infty$ [, we define the open ball $B(x, r, t)$ with center $x \in X$ and radius $r \in L \backslash\left\{0_{\mathcal{L}}, 1_{\mathcal{L}}\right\}$, as

$$
B(x, r, t)=\left\{y \in X: \mathcal{N}(r)<_{L} \mathcal{P}(x-y, t)\right\} .
$$

A subset $A \subseteq X$ is called open if for each $x \in A$, there exist $t>0$ and $r \in L \backslash\left\{0_{\mathcal{L}}, 1_{\mathcal{L}}\right\}$ such that $B(x, r, t) \subseteq A$. Let $\tau_{\mathcal{P}}$ denote the family of all open subsets of $X$. Then $\tau_{\mathcal{P}}$ is called the topology induced by the $\mathcal{L}$-fuzzy norm $\mathcal{P}$.

A subset $A \subseteq X$ is called compact if every open covering has a finite sub-covering. Also a subset $A \subseteq X$ is called closed if from $x_{n} \in A$, for all $n \in \mathbf{N}$, and $x_{n} \stackrel{\mathcal{P}}{\rightarrow} x$ it follows that $x \in A$.

Theorem 4.11 Let $(X, \mathcal{P}, \mathcal{T})$ be an $\mathcal{L}$-fuzzy normed space. A subset $A \subseteq X$ is closed if and only if $X \backslash A$ is open.

Remark 4.12 ([7]) In an $\mathcal{L}$-fuzzy normed space $(X, \mathcal{P}, \mathcal{T})$ whenever $\mathcal{N}(r)<{ }_{L} \mathcal{P}(x, t)$ for $x \in X, t>0$ and $r \in L \backslash\left\{0_{\mathcal{L}}, 1_{\mathcal{L}}\right\}$, we can find a $0<t_{0}<t$ such that $\mathcal{N}(r)<L$

Corollary 4.13 Let $B(x, r, t)$ be an open ball in an $\mathcal{L}$-fuzzy normed space and let $z$ be a member of it. Then there exists $0<t_{0}<t$ such that $z \in B\left(x, r, t_{0}\right)$. 
Definition 4.14 Let $(X, \mathcal{P}, \mathcal{T})$ be an $\mathcal{L}$-fuzzy normed space. A subset $A$ of $X$ is said to be $\mathcal{L} F$-bounded if there exist $t>0$ and $r \in L \backslash\left\{0_{\mathcal{L}}, 1_{\mathcal{L}}\right\}$ such that $\mathcal{N}(r){ }_{L} \mathcal{P}(x, t)$ for each $x \in A$.

Theorem 4.15 In an $\mathcal{L}$-fuzzy normed space every compact set is closed and $\mathcal{L} F$-bounded.

Definition 4.16 Let $X$ and $Y$ be two $\mathcal{L}$-fuzzy normed spaces. A function $g: X \rightarrow Y$ is said to be continuous at a point $x_{0} \in X$ if for any sequence $\left\{x_{n}\right\}$ in $X$ converging to a point $x_{0} \in X$, the sequence $\left\{g\left(x_{n}\right)\right\}$ in $Y$ converges to $g\left(x_{0}\right) \in Y$. If $g$ is continuous at each $x \in X$, then $g: X \rightarrow Y$ is said to be continuous on $X$.

Example 4.17 ([17]) Let $(X,\|\cdot\|)$ be an ordinary normed space and $\phi$ be an increasing and continuous function from $\mathbb{R}^{+}$into $(0,1)$ such that $\lim _{t \rightarrow \infty} \phi(t)=1$. Four typical examples of these functions are as follows:

$$
\begin{aligned}
\phi(t) & =\frac{t}{t+1}, & \phi(t) & =\sin \left(\frac{\pi t}{2 t+1}\right), \\
\phi(t) & =1-e^{-t}, & \phi(t) & =e^{\frac{-1}{t}} .
\end{aligned}
$$

Let $L=[0,1]$ and $\mathcal{T}=\mathbf{M}=\min$. For any $t \in(0, \infty)$, we define

$$
\mathcal{P}(x, t)=[\phi(t)]^{\|x\|}, \quad \forall x \in X,
$$

then $(X, \mathcal{P}, \min )$ is a fuzzy normed space.

Lemma $4.18([22,23])$ Let $(X, \mathcal{P}, \mathbf{M})$ be an $\mathcal{L}$-fuzzy normed space. Let $\left\{x_{n}\right\}$ be a sequence in X. If

$$
\mathcal{P}\left(x_{n+1}-x_{n}, k t\right) \geq_{L} \mathcal{P}\left(x_{n}-x_{n-1}, t\right)
$$

for some $k>1, n \in \mathbb{N}$ and $t>0$, then the sequence $\left\{x_{n}\right\}$ is Cauchy.

Lemma 4.19 Let $(X, \mathcal{P}, \mathbf{M})$ be an $\mathcal{L}$-fuzzy normed space. Define

$$
\mathcal{Q}(x, y, z, t)=\mathbf{M}(\mathcal{P}(x, t), \mathcal{P}(y, t), \mathcal{P}(z, t))
$$

for all $x, y, z \in X$ and $t>0$. Then $\mathcal{Q}$ define an $\mathcal{L}$-fuzzy norm on $X^{3} \times(0, \infty)$.

Proof Let $\mathcal{Q}(x, y, z, t)=1_{\mathcal{L}}$ then $\mathbf{M}(\mathcal{P}(x, t), \mathcal{P}(y, t), \mathcal{P}(z, t))=1_{\mathcal{L}}$, which implies that $x=y=$ $z=0$, the converse is trivial,

$$
\begin{aligned}
\mathcal{Q}(\alpha x, \alpha y, \alpha z, t) & =\mathbf{M}(\mathcal{P}(\alpha x, t), \mathcal{P}(\alpha y, t), \mathcal{P}(\alpha z, t)) \\
& =\mathbf{M}\left(\mathcal{P}\left(x, \frac{t}{\alpha}\right), \mathcal{P}\left(y, \frac{t}{\alpha}\right), \mathcal{P}\left(z, \frac{t}{\alpha}\right)\right) \\
& =\mathcal{Q}\left(x, y, z, \frac{t}{\alpha}\right)
\end{aligned}
$$


for $x, y, z \in X, \alpha \neq 0$ and $t>0$,

$$
\begin{aligned}
\mathcal{Q} & \left(x+x^{\prime}, y+y^{\prime}, z+z^{\prime}, t+s\right) \\
& =\mathbf{M}\left(\mathcal{P}\left(x+x^{\prime}, t+s\right), \mathcal{P}\left(y+y^{\prime}, t+s\right), \mathcal{P}\left(z+z^{\prime}, t+s\right)\right) \\
& \geq \mathbf{M}\left(\mathcal{P}(x, t), \mathcal{P}\left(x^{\prime}, s\right), \mathcal{P}(y, t), \mathcal{P}\left(y^{\prime}, s\right), \mathcal{P}(z, t), \mathcal{P}\left(z^{\prime}, s\right)\right) \\
& =\mathbf{M}\left([\mathcal{P}(x, t), \mathcal{P}(y, t), \mathcal{P}(z, t)],\left[\mathcal{P}\left(x^{\prime}, s\right), \mathcal{P}\left(y^{\prime}, s\right), \mathcal{P}\left(z^{\prime}, s\right)\right]\right) \\
& =\mathbf{M}\left(\mathcal{Q}(x, y, z, t), \mathcal{Q}\left(x^{\prime}, y^{\prime}, z^{\prime}, s\right)\right)
\end{aligned}
$$

for $x, y, z, x^{\prime}, y^{\prime}, z^{\prime} \in X$ and $t, s>0$.

Lemma 4.20 Let $\mathcal{Q}$ be an $\mathcal{L}$-fuzzy norm on $X^{3} \times(0, \infty)$. If

$$
\mathcal{Q}\left(x_{n+1}-x_{n}, y_{n+1}-y_{n}, z_{n+1}-z_{n}, k t\right) \geq_{L} \mathcal{Q}\left(x_{n}-x_{n-1}, y_{n}-y_{n-1}, z_{n}-z_{n-1}, t\right)
$$

for some $k>1, n \in \mathbb{N}$ and $t>0$, then the sequences $\left\{x_{n}\right\},\left\{y_{n}\right\}$, and $\left\{z_{n}\right\}$ are Cauchy.

Proof By Lemmas 4.18 and 4.19 the proof is easy.

Definition 4.21 ([24]) Let $X$ be a non-empty set. An element $(x, y, z) \in X \times X \times X$ is called a tripled fixed point of $F: X \times X \times X \rightarrow X$ if

$$
x=F(x, y, z), \quad y=F(y, x, y) \quad \text { and } \quad z=F(z, y, x) .
$$

Definition 4.22 Let $X$ be a non-empty set. An element $(x, y, z) \in X \times X \times X$ is called a tripled coincidence point of mappings $F: X \times X \times X \rightarrow X$ and $g: X \rightarrow X$ if

$$
g(x)=F(x, y, z), \quad g(y)=F(y, x, y) \quad \text { and } \quad g(z)=F(z, y, x) \text {. }
$$

Definition $4.23([24])$ Let $(X, \preceq)$ be a partially ordered set. A mapping $F: X \times X \times X \rightarrow X$ is said to have the mixed monotone property if $F$ is monotone non-decreasing in its first and third argument and is monotone non-increasing in its second argument, that is, for any $x, y, z \in X$

$$
\begin{aligned}
& x_{1}, x_{2} \in X, \quad x_{1} \preceq x_{2} \quad \Longrightarrow \quad F\left(x_{1}, y, z\right) \preceq F\left(x_{2}, y, z\right), \\
& y_{1}, y_{2} \in X, \quad y_{1} \preceq y_{2} \quad \Longrightarrow \quad F\left(x, y_{2}, z\right) \preceq F\left(x, y_{1}, z\right)
\end{aligned}
$$

and

$$
z_{1}, z_{2} \in X, \quad z_{1} \preceq z_{2} \quad \Longrightarrow \quad F\left(x, y, z_{1}\right) \preceq F\left(x, y, z_{2}\right) .
$$

Definition 4.24 Let $(X, \preceq)$ be a partially ordered set, and $g: X \rightarrow X$. A mapping $F$ : $X \times X \times X \rightarrow X$ is said to have the mixed $g$-monotone property if $F$ is monotone $g$-nondecreasing in its first and third argument and is monotone $g$-non-increasing in its second 
argument, that is, for any $x, y, z \in X$

$$
\begin{aligned}
& x_{1}, x_{2} \in X, \quad g\left(x_{1}\right) \preceq g\left(x_{2}\right) \quad \Longrightarrow \quad F\left(x_{1}, y, z\right) \preceq F\left(x_{2}, y, z\right), \\
& y_{1}, y_{2} \in X, \quad g\left(y_{1}\right) \preceq g\left(y_{2}\right) \quad \Longrightarrow \quad F\left(x, y_{2}, z\right) \preceq F\left(x, y_{1}, z\right)
\end{aligned}
$$

and

$$
z_{1}, z_{2} \in X, \quad g\left(z_{1}\right) \preceq g\left(z_{2}\right) \quad \Longrightarrow \quad F\left(x, y, z_{1}\right) \preceq F\left(x, y, z_{2}\right) .
$$

Lemma 4.25 ([25]) Let $X$ be a non-empty set and $g: X \rightarrow X$ be a mapping. Then there exists a subset $E \subseteq X$ such that $g(E)=g(X)$ and $g: E \rightarrow X$ is one-to-one.

\section{Main results}

Theorem 5.1 Let $(X, \mathcal{P}, \mathbf{M})$ be a complete $\mathcal{L}$-fuzzy normed space, $\preceq$ be a partial order on $X$. Suppose that $F: X \times X \times X \rightarrow X$ has mixed monotone property and

$$
\mathcal{P}(F(x, y, z)-F(u, v, w), k t) \geq_{L} \mathbf{M}(\mathcal{P}(x-u, t), \mathcal{P}(y-v, t), \mathcal{P}(z-w, t))
$$

for all those $x, y, z, u, v, w$ in $X$ for which $x \preceq u, y \succeq v, z \preceq w$,where $0<k<1$. If either

(a) $F$ is continuous or

(b) $X$ has the following properties:

(bi) if $\left\{x_{n}\right\}$ is a non-decreasing sequence and $\lim _{n \rightarrow \infty} x_{n}=x$ then $x_{n} \preceq x$ for all $n \in \mathbb{N}$,

(bii) if $\left\{y_{n}\right\}$ is a non-decreasing sequence and $\lim _{n \rightarrow \infty} y_{n}=y$ then $y_{n} \succeq y$ for all $n \in \mathbb{N}$,

(biii) if $\left\{z_{n}\right\}$ is a non-decreasing sequence and $\lim _{n \rightarrow \infty} z_{n}=y$ then $z_{n} \preceq z$ for all $n \in \mathbb{N}$, then $F$ has a tripled fixed point provided that there exist $x_{0}, y_{0}, z_{0} \in X$ such that

$$
x_{0} \preceq F\left(x_{0}, y_{0}, z_{0}\right), \quad y_{0} \succeq F\left(y_{0}, x_{0}, y_{0}\right), \quad z_{0} \preceq F\left(z_{0}, y_{0}, x_{0}\right) .
$$

Proof Let $x_{0}, y_{0}, z_{0} \in X$ be such that

$$
x_{0} \preceq F\left(x_{0}, y_{0}, z_{0}\right), \quad y_{0} \succeq F\left(y_{0}, x_{0}, y_{0}\right), \quad z_{0} \preceq F\left(z_{0}, y_{0}, x_{0}\right) .
$$

As $F(X \times X \times X) \subseteq X$, so we can construct sequences $\left\{x_{n}\right\},\left\{y_{n}\right\}$ and $\left\{z_{n}\right\}$ in $X$ such that

$$
x_{n+1}=F\left(x_{n}, y_{n}, z_{n}\right), \quad y_{n+1}=F\left(y_{n}, x_{n}, y_{n}\right), \quad z_{n+1}=F\left(z_{n}, y_{n}, x_{n}\right), \quad \forall n \geq 0 .
$$

Now we show that

$$
x_{n} \preceq x_{n+1}, \quad y_{n} \succeq y_{n+1}, \quad z_{n} \preceq z_{n+1}, \quad \forall n \geq 0 .
$$

Since

$$
x_{0} \preceq F\left(x_{0}, y_{0}, z_{0}\right), \quad y_{0} \succeq F\left(y_{0}, x_{0}, y_{0}\right), \quad z_{0} \preceq F\left(z_{0}, y_{0}, x_{0}\right),
$$

(5.3) holds for $n=0$. Suppose that (5.3) holds for any $n \geq 0$. That is,

$$
x_{n} \preceq x_{n+1}, \quad y_{n} \succeq y_{n+1}, \quad z_{n} \preceq z_{n+1} .
$$


As $F$ has the mixed monotone property, by (5.4) we obtain

$$
\left\{\begin{array}{lr}
F\left(x_{n}, y, z\right) \preceq F\left(x_{n+1}, y, z\right), \quad \text { (i) } \\
F\left(x, y_{n}, z\right) \preceq F\left(x, y_{n+1}, z\right), \quad \text { (ii) } \\
F\left(x, y, z_{n}\right) \preceq F\left(x, y, z_{n+1}\right), \quad \text { (iii) }
\end{array}\right.
$$

which on replacing $y$ by $y_{n}$ and $z$ by $z_{n}$ in (i) implies that $F\left(x_{n}, y_{n}, z_{n}\right) \preceq F\left(x_{n+1}, y_{n}, z_{n}\right)$, replacing $x$ by $x_{n+1}$ and $z$ by $z_{n}$ in (ii), we obtain $F\left(x_{n+1}, y_{n}, z_{n}\right) \preceq F\left(x_{n+1}, y_{n+1}, z_{n}\right)$, replacing $y$ by $y_{n+1}$ and $x$ by $x_{n+1}$ in (iii), we get $F\left(x_{n+1}, y_{n+1}, z_{n}\right) \preceq F\left(x_{n+1}, y_{n+1}, z_{n+1}\right)$. Thus we have $F\left(x_{n}, y_{n}, z_{n}\right) \preceq F\left(x_{n+1}, y_{n+1}, z_{n+1}\right)$, that is, $x_{n+1} \preceq x_{n+2}$. Similarly, we have

$$
\left\{\begin{array}{lr}
F\left(y, x, y_{n+1}\right) \preceq F\left(y, x, y_{n}\right), & \text { (iv) } \\
F\left(y_{n+1}, x, y\right) \preceq F\left(y_{n}, x, y\right), & \quad(\mathrm{v}) \\
F\left(y, x_{n+1}, y\right) \preceq F\left(y, x_{n}, y\right), & \quad(\mathrm{vi})
\end{array}\right.
$$

which on replacing $y$ by $y_{n+1}$ and $x$ by $x_{n+1}$ in (iv) implies that $F\left(y_{n+1}, x_{n+1}, y_{n+1}\right) \preceq$ $F\left(y_{n+1}, x_{n+1}, y_{n}\right)$, replacing $x$ by $x_{n+1}$ and $y$ by $y_{n+1}$ in (v), we obtain $F\left(y_{n+1}, x_{n+1}, y_{n}\right) \preceq$ $F\left(y_{n}, x_{n+1}, y_{n}\right)$, replacing $y$ by $y_{n}$ in (vi), we get $F\left(y_{n}, x_{n+1}, y_{n}\right) \preceq F\left(y_{n}, x_{n}, y_{n}\right)$. Thus we have $F\left(y_{n+1}, x_{n+1}, y_{n+1}\right) \preceq F\left(y_{n}, x_{n}, y_{n}\right)$, that is, $y_{n+2} \preceq y_{n+1}$. Similarly, we have

$$
\left\{\begin{array}{rrr}
F\left(z_{n}, y, x\right) \preceq F\left(z_{n+1}, y, x\right), & \quad \text { (vii) } \\
F\left(z, y_{n}, x\right) \preceq F\left(z, y_{n+1}, x\right), & \quad \text { (viii) } \\
F\left(z, y, x_{n}\right) \preceq F\left(z, y, x_{n+1}\right), & \quad \text { (xi) }
\end{array} \mid\right.
$$

which on replacing $y$ by $y_{n}$ and $x$ by $x_{n}$ in (vii) implies that $F\left(z_{n}, y_{n}, x_{n}\right) \preceq F\left(z_{n+1}, y_{n}, x_{n}\right)$, replacing $x$ by $x_{n}$ and $z$ by $z_{n+1}$ in (viii), we obtain $F\left(z_{n+1}, y_{n}, x_{n}\right) \preceq F\left(z_{n+1}, y_{n+1}, x_{n}\right)$, replacing $y$ by $y_{n+1}$ and $z$ by $z_{n+1}$ in (xi), we get $F\left(z_{n+1}, y_{n+1}, x_{n}\right) \preceq F\left(z_{n+1}, y_{n+1}, x_{n+1}\right)$. Thus we have $F\left(z_{n}, y_{n}, x_{n}\right) \preceq F\left(z_{n+1}, y_{n+1}, x_{n+1}\right)$, that is, $z_{n+1} \preceq z_{n+2}$. So by induction, we conclude that (5.4) holds for all $n \geq 0$, that is

$$
\begin{aligned}
& x_{0} \preceq x_{1} \preceq x_{2} \preceq \cdots \preceq x_{n} \preceq x_{n+1} \preceq \cdots, \\
& y_{0} \succeq y_{1} \succeq y_{2} \succeq \cdots \succeq y_{n} \succeq y_{n+1} \succeq \cdots, \\
& z_{0} \preceq z_{1} \preceq z_{2} \preceq \cdots \preceq z_{n} \preceq z_{n+1} \preceq \cdots .
\end{aligned}
$$

Consider

$$
\begin{aligned}
\mathcal{P}\left(x_{n}-x_{n+1}, k t\right) & =\mathcal{P}\left(F\left(x_{n-1}, y_{n-1}, z_{n-1}\right)-F\left(x_{n}, y_{n}, z_{n}\right), k t\right) \\
& \geq_{L} \mathbf{M}\left(\mathcal{P}\left(x_{n-1}-x_{n}, t\right), \mathcal{P}\left(y_{n-1}-y_{n}, t\right), \mathcal{P}\left(z_{n-1}-z_{n}, t\right)\right) \\
& =\mathcal{Q}\left(x_{n-1}-x_{n}, y_{n-1}-y_{n}, z_{n-1}-z_{n}, t\right) .
\end{aligned}
$$

Also,

$$
\begin{aligned}
\mathcal{P}\left(z_{n}-z_{n+1}, k t\right) & =\mathcal{P}\left(F\left(z_{n-1}, y_{n-1}, x_{n-1}\right)-F\left(z_{n}, y_{n}, x_{n}\right), k t\right) \\
& \geq_{L} \mathbf{M}\left(\mathcal{P}\left(z_{n-1}-z_{n}, t\right), \mathcal{P}\left(y_{n-1}-y_{n}, t\right), \mathcal{P}\left(x_{n-1}-x_{n}, t\right)\right)
\end{aligned}
$$




$$
\begin{aligned}
& =\mathbf{M}\left(\mathcal{P}\left(x_{n-1}-x_{n}, t\right), \mathcal{P}\left(y_{n-1}-y_{n}, t\right), \mathcal{P}\left(z_{n-1}-z_{n}, t\right)\right) \\
& =\mathcal{Q}\left(x_{n-1}-x_{n}, y_{n-1}-y_{n}, z_{n-1}-z_{n}, t\right) .
\end{aligned}
$$

Now,

$$
\begin{array}{rl}
\mathcal{P}\left(y_{n}-y_{n+1}, k t\right)= & \mathcal{P}\left(F\left(y_{n-1}, x_{n-1}, y_{n-1}\right)-F\left(y_{n}, x_{n}, y_{n}\right), k t\right) \\
\geq_{L} \mu\left(y_{n-1}-y_{n}, t\right) * \mu\left(x_{n-1}-x_{n}, t\right) * \mu\left(y_{n-1}-y_{n}, t\right) \\
= & \mathbf{M}\left(\mathcal{P}\left(y_{n-1}-y_{n}, t\right), \mathcal{P}\left(x_{n-1}-x_{n}, t\right), \mathcal{P}\left(y_{n-1}-y_{n}, t\right)\right) \\
\geq_{L} & \mathbf{M}\left(\mathcal{P}\left(y_{n-1}-y_{n}, t\right), \mathcal{P}\left(x_{n-1}-x_{n}, t\right), \mathcal{P}\left(y_{n-1}-y_{n}, t\right),\right. \\
& \left.\mathcal{P}\left(z_{n-1}-z_{n}, t\right), \mathcal{P}\left(z_{n-1}-z_{n}, t\right), \mathcal{P}\left(x_{n-1}-x_{n}, t\right)\right) \\
\geq_{L} & \mathcal{Q}\left(x_{n-1}-x_{n}, y_{n-1}-y_{n}, z_{n-1}-z_{n}, t\right) .
\end{array}
$$

By (5.8)-(5.10), we obtain

$$
\mathcal{Q}\left(x_{n}-x_{n+1}, y_{n}-y_{n+1}, z_{n}-z_{n+1}, k t\right) \geq_{L} \mathcal{Q}\left(x_{n-1}-x_{n}, y_{n-1}-y_{n}, z_{n-1}-z_{n}, t\right)
$$

for $t>0$. By Lemma 4.20 we conclude that $\left\{x_{n}\right\},\left\{y_{n}\right\}$, and $\left\{z_{n}\right\}$ are Cauchy sequences in $X$. Since $X$ is complete, there exist $x, y$, and $z$ such that $\lim _{n \rightarrow \infty} x_{n}=x, \lim _{n \rightarrow \infty} y_{n}=y$, and $\lim _{n \rightarrow \infty} z_{n}=z$. If the assumption (a) does hold, then we have

$$
\begin{aligned}
x & =\lim _{n \rightarrow \infty} x_{n+1}=\lim _{n \rightarrow \infty} F\left(x_{n}, y_{n}, z_{n}\right) \\
& =F\left(\lim _{n \rightarrow \infty} x_{n}, \lim _{n \rightarrow \infty} y_{n}, \lim _{n \rightarrow \infty} z_{n}\right)=F(x, y, z), \\
y & =\lim _{n \rightarrow \infty} y_{n+1}=\lim _{n \rightarrow \infty} F\left(y_{n}, x_{n}, y_{n}\right) \\
& =F\left(\lim _{n \rightarrow \infty} y_{n}, \lim _{n \rightarrow \infty} x_{n}, \lim _{n \rightarrow \infty} y_{n}\right)=F(y, x, y)
\end{aligned}
$$

and

$$
\begin{aligned}
z & =\lim _{n \rightarrow \infty} z_{n+1}=\lim _{n \rightarrow \infty} F\left(z_{n}, y_{n}, x_{n}\right) \\
& =F\left(\lim _{n \rightarrow \infty} z_{n}, \lim _{n \rightarrow \infty} y_{n}, \lim _{n \rightarrow \infty} x_{n}\right)=F(z, y, x) .
\end{aligned}
$$

Suppose that assumption (b) holds then

$$
\begin{aligned}
\mathcal{P} & \left(x_{n+1}-F(x, y, z), k t\right) \\
& =\mathcal{P}\left(F\left(x_{n}, y_{n}, z_{n}\right)-F(x, y, z), k t\right) \geq_{L} \mathcal{P}\left(x_{n}-x, y_{n}-y, z_{n}-z, t\right),
\end{aligned}
$$

which, on taking the limit as $n \rightarrow \infty$, gives $\mathcal{P}(x-F(x, y, z), k t)=1_{\mathcal{L}}, x=F(x, y, z)$. Also,

$$
\begin{aligned}
& \mathcal{P}\left(y_{n+1}-F(y, x, y), k t\right) \\
& \quad=\mathcal{P}\left(F\left(y_{n}, x_{n}, y_{n}\right)-F(y, x, y), k t\right) \geq_{L} \mathcal{P}\left(y_{n}-y, x_{n}-x, y_{n}-y, t\right),
\end{aligned}
$$


which on taking the limit as $n \rightarrow \infty$, implies $\mathcal{P}(y-F(y, x, y), k t)=1_{\mathcal{L}}, y=F(y, x, y)$. Finally, we have

$$
\begin{aligned}
\mathcal{P} & \left(z_{n+1}-F(z, y, x), k t\right) \\
& =\mathcal{P}\left(F\left(z_{n}, y_{n}, x_{n}\right)-F(z, y, x), k t\right) \geq_{L} \mathcal{P}\left(z_{n}-z, y_{n}-y, x_{n}-x, t\right),
\end{aligned}
$$

which on taking the limit as $n \rightarrow \infty$, gives $\mathcal{P}(z-F(z, y, x), k t)=1_{\mathcal{L}}, z=F(z, y, x)$.

Theorem 5.2 Let $(X, \mathcal{P}, \mathbf{M})$ be a complete $\mathcal{L}$-fuzzy normed space, $\preceq$ be a partial order on $X$. Let $F: X \times X \times X \rightarrow X$, and $g: X \rightarrow X$ be mappings such that $F$ has a mixed $g$-monotone property and

$$
\begin{aligned}
& \mathcal{P}(F(x, y, z)-F(u, v, w), k t) \\
& \quad{ }_{L} \mathbf{M}(\mathcal{P}(g x-g u, t), \mathcal{P}(g y-g v, t), \mathcal{P}(g z-g w, t))
\end{aligned}
$$

for all those $x, y, z$, and $u, v, w$ for which $g x \preceq g u, g y \succeq g v, g z \preceq g w$, where $0<k<1$. Assume that $g(X)$ is complete, $F(X \times X \times X) \subseteq g(X)$ and $g$ is continuous. If either

(a) $F$ is continuous or

(b) $X$ has the following properties:

(bi) if $\left\{x_{n}\right\}$ is a non-decreasing sequence and $\lim _{n \rightarrow \infty} x_{n}=x$ then $x_{n} \preceq x$ for all $n \in \mathbb{N}$

(bii) if $\left\{y_{n}\right\}$ is a non-decreasing sequence and $\lim _{n \rightarrow \infty} y_{n}=y$ then $y_{n} \succeq y$ for all $n \in \mathbb{N}$, and

(biii) if $\left\{z_{n}\right\}$ is a non-decreasing sequence and $\lim _{n \rightarrow \infty} z_{n}=y$ then $z_{n} \preceq z$ for all $n \in \mathbb{N}$, then $F$ has a tripled coincidence point provided that there exist $x_{0}, y_{0}, z_{0} \in X$ such that

$$
g\left(x_{0}\right) \preceq F\left(x_{0}, y_{0}, z_{0}\right), \quad g\left(y_{0}\right) \succeq F\left(y_{0}, x_{0}, y_{0}\right), \quad g\left(z_{0}\right) \preceq F\left(z_{0}, y_{0}, x_{0}\right) .
$$

Proof By Lemma 4.25, there exists $E \subseteq X$ such that $g: E \rightarrow X$ is one-to-one and $g(E)=$ $g(X)$. Now define a mapping $\mathcal{A}: g(E) \times g(E) \times g(E) \rightarrow X$, by

$$
\mathcal{A}(g x, g y, g z)=F(x, y, z), \quad \forall x, y, z \in X .
$$

Since $g$ is one-to-one, so $\mathcal{A}$ is well defined. Now, (5.11) and (5.12) implies that

$$
\begin{aligned}
& \mathcal{P}(\mathcal{A}(g x, g y, g z)-\mathcal{A}(g u, g v, g w), k t) \\
& \quad \geq_{L} \mathbf{M}(\mathcal{P}(g x-g u, t), \mathcal{P}(g y-g v, t), \mathcal{P}(g z-g w, t))
\end{aligned}
$$

for all $x, y, z, u, v, w \in E$ for which $g x \preceq g u, g y \succeq g v, g z \preceq g w$. Since $F$ has a mixed $g$-monotone property for all $x, y, z \in X$, so we have

$$
\begin{array}{ll}
x_{1}, x_{2} \in X, & g\left(x_{1}\right) \preceq g\left(x_{2}\right) \quad \Longrightarrow \quad F\left(x_{1}, y, z\right) \preceq F\left(x_{2}, y, z\right), \\
y_{1}, y_{2} \in X, & g\left(y_{1}\right) \preceq g\left(y_{2}\right) \quad \Longrightarrow \quad F\left(x, y_{2}, z\right) \preceq F\left(x, y_{1}, z\right) \quad \text { and } \\
z_{1}, z_{2} \in X, \quad g\left(z_{1}\right) \preceq g\left(z_{2}\right) \quad \Longrightarrow \quad F\left(x, y, z_{1}\right) \preceq F\left(x, y, z_{2}\right) .
\end{array}
$$


Now, from (5.12) and (5.14) we have

$$
\begin{array}{ll}
x_{1}, x_{2} \in X, \quad g\left(x_{1}\right) \preceq g\left(x_{2}\right) \quad \Longrightarrow & \mathcal{A}\left(g x_{1}, g y, g z\right) \preceq \mathcal{A}\left(g x_{2}, g y, g z\right), \\
y_{1}, y_{2} \in X, & g\left(y_{1}\right) \preceq g\left(y_{2}\right) \quad \Longrightarrow \quad \mathcal{A}\left(g x, g y_{2}, g z\right) \preceq \mathcal{A}\left(g x, g y_{1}, g z\right), \\
z_{1}, z_{2} \in X, \quad g\left(z_{1}\right) \preceq g\left(z_{2}\right) \quad \Longrightarrow & \mathcal{A}\left(g x, g y, g z_{1}\right) \preceq \mathcal{A}\left(g x, g y, g z_{2}\right) .
\end{array}
$$

Hence $\mathcal{A}$ has a mixed monotone property. Suppose that assumption (a) holds. Since $F$ is continuous, $\mathcal{A}$ is also continuous. By using Theorem 5.1, $\mathcal{A}$ has a tripled fixed point $(u, v, w) \in g(E) \times g(E) \times g(E)$. If assumption (b) holds, then using the definition of $\mathcal{A}$, following similar arguments to those given in Theorem 5.1, $\mathcal{A}$ has a tripled fixed point $(u, v, w) \in g(E) \times g(E) \times g(E)$. Finally, we show that $F$ and $g$ have tripled coincidence point. Since $\mathcal{A}$ has a tripled fixed point $(u, v, w) \in g(E) \times g(E) \times g(E)$ we get

$$
u=\mathcal{A}(u, v, w), \quad v=\mathcal{A}(v, u, v), \quad w=\mathcal{A}(w, u, v) .
$$

Hence, there exist $u_{1}, v_{1}, w_{1} \in X \times X \times X$ such that $g u_{1}=u, g v_{1}=v$, and $g w_{1}=w$. Now, it follows from (5.16) that

$$
\begin{aligned}
& g u_{1}=\mathcal{A}\left(g u_{1}, g v_{1}, w\right)=F\left(u_{1}, v_{1}, w_{1}\right), \\
& g v_{1}=\mathcal{A}\left(g v_{1}, g u_{1}, g v_{1}\right)=F\left(v_{1}, u_{1}, v_{1}\right) \quad \text { and } \\
& g w_{1}=\mathcal{A}\left(g w_{1}, g u_{1}, g v_{1}\right)=F\left(w_{1}, v_{1}, u_{1}\right) .
\end{aligned}
$$

Thus $\left(u_{1}, v_{1}, w_{1}\right) \in X \times X \times X$ is a tripled coincidence point of $F$ and $g$.

Example 5.3 ([17]) Let $X=\mathbb{R}$. Consider Example 4.17 and let $\phi: \mathbb{R}^{+} \rightarrow(0,1)$ be defined by $\phi(t)=e^{-\frac{1}{t}}$ for all $t \in \mathbb{R}^{+}$. Then

$$
\mathcal{P}(x, t)=[\phi(t)]^{|x|}
$$

for all $x \in X$ and $t>0$.

If $X$ is endowed with the usual order as $x \preceq y \Longleftrightarrow x-y \leq 0$, then $(X, \preceq)$ is a partially ordered set. Define mappings $F: X \times X \times X \rightarrow X$, and $g: X \rightarrow X$ by

$$
F(x, y, z)=2 x-2 y+2 z+1 \text { and } g(x)=7 x-1 .
$$

Obviously, $F$ and $g$ both are onto maps, so $F(X \times X \times X) \subseteq g(X)$, also $F$ and $g$ are continuous and $F$ has the mixed $g$-monotone property. Indeed,

$$
\begin{aligned}
x_{1}, x_{2} \in X, \quad g x_{1} \preceq g x_{2} & \Longrightarrow \quad 2 x_{1}-2 y+2 z+1 \leq 2 x_{2}-2 y+2 z+1 \\
& \Longrightarrow \quad F\left(x_{1}, y, z\right) \preceq F\left(x_{2}, y, z\right) .
\end{aligned}
$$

Similarly, we can prove that

$$
y_{1}, y_{2} \in X, \quad g\left(y_{1}\right) \preceq g\left(y_{2}\right) \quad \Longrightarrow \quad F\left(x, y_{2}, z\right) \preceq F\left(x, y_{1}, z\right)
$$


and

$$
z_{1}, z_{2} \in X, \quad g\left(z_{1}\right) \preceq g\left(z_{2}\right) \quad \Longrightarrow \quad F\left(x, y, z_{1}\right) \preceq F\left(x, y, z_{2}\right) .
$$

If, $x_{0}=0, y_{0}=\frac{2}{3}, z_{0}=0$, then

$$
\begin{aligned}
& -1=g\left(x_{0}\right) \preceq F\left(x_{0}, y_{0}, z_{0}\right)=-\frac{1}{3}, \\
& \frac{11}{3}=g\left(y_{0}\right) \succeq F\left(y_{0}, x_{0}, y_{0}\right)=\frac{11}{3}, \\
& -1=g\left(z_{0}\right) \preceq F\left(z_{0}, y_{0}, x_{0}\right)=-\frac{1}{3} .
\end{aligned}
$$

So there exist $x_{0}, y_{0}, z_{0} \in X$ such that

$$
g\left(x_{0}\right) \preceq F\left(x_{0}, y_{0}, z_{0}\right), \quad g\left(y_{0}\right) \succeq F\left(y_{0}, x_{0}, y_{0}\right), \quad g\left(z_{0}\right) \preceq F\left(z_{0}, y_{0}, x_{0}\right) .
$$

Now for all $x, y, z, u, v, w \in X$, for which $g x \preceq g u, g y \succeq g v, g z \preceq g w$, we have

$$
\begin{aligned}
\min & (\mathcal{P}(g x-g u, t), \mathcal{P}(g y-g v, t), \mathcal{P}(g z-g w, t)) \\
& =\min (\mathcal{P}(7(x-u), t), \mathcal{P}(7(y-v), t), \mathcal{P}(7(z-w), t)) \\
& =\min \left(\mathcal{P}\left((x-u), \frac{t}{7}\right), \mathcal{P}\left((y-v), \frac{t}{7}\right), \mathcal{P}\left((z-w), \frac{t}{7}\right)\right) \\
& =\min \left(\mathcal{P}\left((x-u), \frac{t}{7}\right), \mathcal{P}\left((v-y), \frac{t}{7}\right), \mathcal{P}\left((z-w), \frac{t}{7}\right)\right) \\
& \leq \mathcal{P}\left(x-u+v-y+z-w, \frac{3 t}{7}\right) \\
& =\left(e^{-\frac{7}{3 t}}\right)^{|(x-u+v-y+z-w)|} \\
& =\left(e^{-\frac{3.5}{3 t}}\right)^{|2(x-u+v-y+z-w)|} \\
& =\left(e^{-\frac{3.5}{3 t}}\right)^{|2(x-u)+2(v-y)+2(z-w)|} \\
& =\left(e^{-\frac{3.5}{3 t}}\right)^{|F(x, y, z)-F(u, v, w)|} \\
& =\mathcal{P}(F(x, y, z)-F(u, v, w), k t)
\end{aligned}
$$

for $k=\frac{3}{3.5}<1$. Hence there exists $k=\frac{3}{3.5}<1$ such that

$$
\mathcal{P}(F(x, y, z)-F(u, v, w), k t) \geq \min (\mathcal{P}(g x-g u, t),(g y-g v, t),(g z-g w, t))
$$

for all $x, y, z, u, v, w \in X$, for which $g x \preceq g u, g y \succeq g v, g z \preceq g w$.

Therefore all the conditions of Theorem 5.2 are satisfied. So $F$ and $g$ have a tripled coincidence point and here $\left(\frac{2}{5}, \frac{2}{5}, \frac{2}{5}\right)$ is a tripled coincidence point of $F$ and $g$.

\section{Application}

In this section, we study the existence of a unique solution to an initial value problem, as an application to the our tripled fixed point theorem. 
Consider the initial value problem

$$
x^{\prime}(\ell)=f(\ell, x(\ell), x(\ell), x(\ell)), \quad \ell \in I=[0,1], \quad x(0)=x_{0},
$$

where $f: I \times \mathbb{R} \times \mathbb{R} \times \mathbb{R} \rightarrow \mathbb{R}$ and $x_{0} \in \mathbb{R}$.

An element $(\alpha, \beta, \gamma) \in C(I, \mathbb{R})^{3}$ is called a tripled initial value problem (6.1) if

$$
\begin{aligned}
& \alpha^{\prime}(\ell) \leq f(\ell, \alpha(\ell), \beta(\ell), \gamma(\ell)), \\
& \beta^{\prime}(\ell) \geq f(\ell, \beta(\ell), \alpha(\ell), \beta(\ell)), \\
& \delta^{\prime}(\ell) \leq f(\ell, \gamma(\ell), \beta(\ell), \alpha(\ell))
\end{aligned}
$$

for each $\ell \in I$ together with the initial condition

$$
\alpha(0)=\beta(0)=\gamma(0)=x_{0} .
$$

Theorem 6.1 Let $(C(I, \mathbb{R}), \mathcal{P}, \mathbf{M})$ be a complete $\mathcal{L}$-fuzzy normed space with the following order relation on $C(I, \mathbb{R})$ :

$$
x, y \in C(I, \mathbb{R}), \quad x \leq y \quad \Longleftrightarrow \quad x(\ell) \leq y(\ell), \quad \forall \ell \in[0,1],
$$

and fuzzy norm

$$
\mathcal{P}(x-y, t)=\inf _{\ell \in I} \frac{t}{t+|x(\ell)-y(\ell)|}, \quad x, y \in C(I, \mathbb{R}), t>0 .
$$

Consider the initial value problem (6.1) with $f \in C\left(I \times \mathbb{R}^{3}, \mathbb{R}\right)$ which is non-decreasing in the second and fourth variables and non-increasing in the third variable. Suppose that for $x \geq u, y \leq v$, and $z \geq w$, we have

$$
0 \leq f(\ell, x, y, z)-f(\ell, u, v, w) \leq k[(x-u)+(v-y)+(z-w)]
$$

where $k \in\left(0, \frac{1}{3}\right)$. Then the existence of a tripled solution for (6.1) provides the existence of a unique solution of $(6.1)$ in $C(I, \mathbb{R})$.

Proof The initial value problem (6.1) is equivalent to the integral equation

$$
x(\ell)=x_{0}+\int_{0}^{\ell} f(s, x(s), x(s), x(s)) d s, \quad \ell \in I .
$$

Suppose $\left\{x_{n}\right\}$ is a non-decreasing sequence in $C(I, \mathbb{R})$ that converges to $x \in C(I, \mathbb{R})$. Then, for every $\ell \in I$, the sequence of the real numbers

$$
x_{1}(\ell) \leq x_{2}(\ell) \leq \cdots \leq x_{n}(\ell) \leq \cdots
$$

converges to $x(\ell)$. Therefore, for all $\ell \in I$ and $n \in \mathbb{N}$, we have $x_{n}(\ell) \leq x(\ell)$. Hence $x_{n} \leq x$ for all $n \in \mathbb{N}$. Also, $C(I, \mathbb{R}) \times C(I, \mathbb{R}) \times C(I, \mathbb{R})$ is a partially ordered set if we define the 
following order relation in $X \times X \times X$ :

$$
(x, y, z) \leq(u, v, w) \quad \Longleftrightarrow \quad x(\ell) \leq u(\ell), \quad v(\ell) \leq y(\ell) \quad \text { and } \quad z(\ell) \leq w(\ell), \quad \forall \ell \in I .
$$

Define $F: C(I, \mathbb{R}) \times C(I, \mathbb{R}) \times C(I, \mathbb{R}) \rightarrow C(I, \mathbb{R})$ by

$$
F(x, y, z)(\ell)=x_{0}+\int_{0}^{\ell} f(s, x(s), y(s), z(s)) d s, \quad \ell \in I .
$$

Now, for $u \leq x, y \leq v$ and $w \leq z$, we have

$$
\begin{aligned}
\mathcal{P} & (F(x, y, z)-F(u, v, w), t) \\
& =\inf _{\ell \in I} \frac{t}{t+\int_{0}^{\ell}[f(s, x(s), y(s), z(s))-f(s, u(s), v(s), w(s))] d s} \\
& \geq \inf _{\ell \in I} \frac{t}{t+\int_{0}^{\ell} k[(x-u)+(v-y)+(z-w)] d s} \\
& \geq \inf _{\ell \in I} \mathcal{M}\left(\frac{\frac{t}{3}}{\frac{t}{3}+\int_{0}^{\ell} k(x-u) d s}, \frac{\frac{t}{3}}{\frac{t}{3}+\int_{0}^{\ell} k(v-y) d s}, \frac{\frac{t}{3}}{\frac{t}{3}+\int_{0}^{\ell} k(z-w) d s}\right) \\
& =\mathcal{M}\left(\mathcal{P}\left(x-u, \frac{t}{3 k}\right), \mathcal{P}\left(y-v, \frac{t}{3 k}\right), \mathcal{P}\left(z-w, \frac{t}{3 k}\right)\right) ;
\end{aligned}
$$

hence

$$
\mathcal{P}(F(x, y, z)-F(u, v, w), 3 k t) \geq \mathcal{M}(\mathcal{P}(x-u, t), \mathcal{P}(y-v, t), \mathcal{P}(z-w, t))
$$

Then $F$ satisfies the condition (5.1) of Theorem 5.1. Now, let $(\alpha, \beta, \gamma)$ be a tripled solution of the initial value problem (6.1); then we have $\alpha \leq F(\alpha, \beta, \gamma), F(\beta, \alpha, \beta) \leq \beta$ and $\gamma \leq F(\gamma, \beta, \alpha)$. Then Theorem 5.1 shows that $F$ has a unique tripled fixed point.

\section{Competing interests}

The authors declare that they have no competing interests.

\section{Authors' contributions}

All authors contributed equally to the writing of this paper. All authors read and approved the final manuscript.

\section{Author details}

${ }^{1}$ Department of Mathematics, Iran University of Science and Technology, Tehran, Iran. ${ }^{2}$ Department of Mathematics, Faculty of Science, King Mongkut's University of Technology Thonburi (KMUTT), Bang Mod, Thrung Khru, Bangkok, 10140 Thailand. ${ }^{3}$ Department of Mathematics, University of Ulsan, Ulsan, 680-749, South Korea.

Received: 1 January 2014 Accepted: 23 May 2014 Published: 03 Jun 2014

\section{References}

1. Saadati, R, Park, JH: On the intuitionistic fuzzy topological spaces. Chaos Solitons Fractals 27(2), 331-344 (2006)

2. Atanassov, KT: Intuitionistic fuzzy sets. Fuzzy Sets Syst. 20(1), 87-96 (1986)

3. Saadati, R, Vaezpour, SM: Some results on fuzzy Banach spaces. J. Appl. Math. Comput. 17(1-2), 475-484 (2005)

4. Saadati, R, Park, C: A note on IFN-spaces. Adv. Differ. Equ. 2014, 63 (2014)

5. Deschrijver, G, O'Regan, D, Saadati, R, Vaezpour, SM: $\mathcal{L}$-Fuzzy Euclidean normed spaces and compactness. Chaos Solitons Fractals 42(1), 40-45 (2009)

6. Zadeh, LA: Fuzzy sets. Inf. Control 8, 338-353 (1965)

7. George, A, Veeramani, P: On some results in fuzzy metric spaces. Fuzzy Sets Syst. 64(3), 395-399 (1994)

8. Kramosil, I, Michalek, J: Fuzzy metric and statistical metric spaces. Kybernetika 11, 326-333 (1975)

9. Grabiec, M: Fixed points in fuzzy metric spaces. Fuzzy Sets Syst. 27(3), 385-389 (1988) 
10. Fang, J: On fixed point theorems in fuzzy metric spaces. Fuzzy Sets Syst. 46, 107-113 (1992)

11. Cho, Y: Fixed points in fuzzy metric spaces. J. Fuzzy Math. 5(4), 949-962 (1997)

12. Gregori, V, Sapena, A: On fixed-point theorems in fuzzy metric spaces. Fuzzy Sets Syst. 125(2), 245-252 (2002)

13. Beg, I, Abbas, M: Common fixed points of Banach operator pair on fuzzy normed spaces. Fixed Point Theory 12(2), 285-292 (2011)

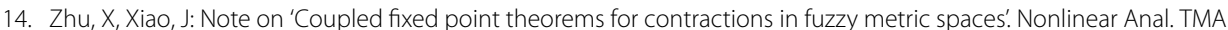
74(16), 5475-5479 (2011)

15. Hu, X: Common coupled fixed point theorems for contractive mappings in fuzzy metric spaces. Fixed Point Theory Appl. 2011, Article ID 363716 (2011)

16. Roldán, A, Martínez-Moreno, J, Roldán, C: Tripled fixed point theorem in fuzzy metric spaces and applications. Fixed Point Theory Appl. 2013, 29 (2013)

17. Abbas, M, Ali, B, Sintunavarat, W, Kumam, P: Tripled fixed point and tripled coincidence point theorems in intuitionistic fuzzy normed spaces. Fixed Point Theory Appl. 2012, 187 (2012)

18. Goguen, JA: L-Fuzzy sets. J. Math. Anal. Appl. 18, 145-174 (1967)

19. Deschrijver, G, Kerre, EE: On the relationship between some extensions of fuzzy set theory. Fuzzy Sets Syst. 133(2) 227-235 (2003)

20. Deschrijver, G, Cornelis, C, Kerre, EE: On the representation of intuitionistic fuzzy $t$-norms and $t$-conorms. Proceedings of the Sixth International Conference on Intuitionistic Fuzzy Set (Varna, 2002). Notes IFS 8, 1-10 (2002)

21. Saadati, R: A note on 'Some results on the IF-normed spaces'. Chaos Solitons Fractals 41(1), 206-213 (2009)

22. Hadžić, O, Pap, E: Fixed Point Theory in Probabilistic Metric Spaces. Mathematics and Its Applications, vol. 536. Kluwer Academic, Dordrecht (2001)

23. Shakeri, S, Ćirić, LJB, Saadati, R: Common fixed point theorem in partially ordered $\mathcal{L}$-fuzzy metric spaces. Fixed Point Theory Appl. 2010, Article ID 125082 (2010)

24. Berinde, $V$, Borcut, $M$ : Tripled fixed point theorems for contractive type mappings in partially ordered metric spaces. Nonlinear Anal. 74(15), 4889-4897 (2011)

25. Haghi, RH, Rezapour, S, Shahzad, N: Some fixed point generalizations are not real generalizations. Nonlinear Anal. 74(5), 1799-1803 (2011)

10.1186/1687-1812-2014-136

Cite this article as: Saadati et al.: On the tripled fixed point and tripled coincidence point theorems in fuzzy normed spaces. Fixed Point Theory and Applications 2014, 2014:136

\section{Submit your manuscript to a SpringerOpen ${ }^{\ominus}$ journal and benefit from:}

- Convenient online submission

- Rigorous peer review

- Immediate publication on acceptance

- Open access: articles freely available online

- High visibility within the field

- Retaining the copyright to your article 